\title{
Photometric and Thermal Cross-calibration of Solar EUV Instruments
}

\author{
P.F. Boerner • P. Testa $\cdot$ H. Warren • M.A. Weber • \\ C.J. Schrijver
}

Received: 11 February 2013 / Accepted: 7 November 2013 / Published online: 7 December 2013

(C) The Author(s) 2013. This article is published with open access at Springerlink.com

\begin{abstract}
We present an assessment of the accuracy of the calibration measurements and atomic physics models that go into calculating the SDO/AIA response as a function of wavelength and temperature. The wavelength response is tested by convolving SDO/EVE and Hinode/EIS spectral data with the AIA effective area functions and by comparing the predictions with AIA observations. For most channels, the AIA intensities summed over the disk agree with the corresponding measurements derived from the current version (V2) of the EVE data to within the estimated $25 \%$ calibration error. This agreement indicates that the AIA effective areas are generally stable in time. The AIA $304 \AA$ channel, however, does show degradation by a factor of almost 3 from May 2010 through September 2011, when the throughput apparently reached a minimum. We also found some inconsistencies in the 335 $\AA$ passband, possibly due to higher-order contamination of the EVE data. The intensities in the AIA $193 \AA$ channel agree to within the uncertainties with the corresponding measurements from EIS full CCD observations. Analysis of high-resolution X-ray spectra of the solar-like corona of Procyon and of EVE spectra allowed us to investigate the accuracy and completeness of the CHIANTI database in the AIA shorter wavelength passbands. We
\end{abstract}

\footnotetext{
P.F. Boerner $(\varangle) \cdot$ C.J. Schrijver

Lockheed Martin Solar and Astrophysics Laboratory, A021S, Bldg 252, 3251 Hanover Street, Palo

Alto, CA 94304, USA

e-mail: boerner@Imsal.com

C.J. Schrijver

e-mail: schrijver@Imsal.com

P. Testa $\cdot$ M.A. Weber

Harvard Smithsonian Center for Astrophysics, 60 Garden Street, Cambridge, MA 02138, USA

P. Testa

e-mail: ptesta@cfa.harvard.edu

M.A. Weber

e-mail: mweber@cfa.harvard.edu

H. Warren

Naval Research Laboratory, 4555 Overlook Ave. S.W., Washington, DC 20375, USA

e-mail: harry.warren@nrl.navy.mil
} 
found that in the $94 \AA$ channel, the spectral model significantly underestimates the plasma emission owing to a multitude of missing lines. We derived an empirical correction for the AIA temperature responses by performing differential emission measure (DEM) inversion on a broad set of EVE spectra and adjusting the AIA response functions so that the count rates predicted by the full-disk DEMs match the observations.

Keywords Atomic data $\cdot$ Chromosphere · Corona $\cdot$ EUV $\cdot$ Instrumentation · Transition region

\section{Introduction}

The Atmospheric Imaging Assembly (AIA; Lemen et al., 2012) onboard the Solar Dynamics Observatory (SDO) is an array of telescopes that continuously observes the full solar disk in nine UV/EUV wavelength channels with high cadence (12 s for EUV channels and $24 \mathrm{~s}$ for UV) and spatial resolution ( $4096 \times 4096$ pixels of 0.6 arcsec each). Its images have facilitated new understanding of numerous phenomena in solar physics, including the global structure of the magnetic field (Schrijver et al., 2011), new types of waves associated with flares (Liu et al., 2011), and the heating of active-region loops (Warren, Brooks, and Winebarger, 2011).

Like earlier instruments such as the EUV Imaging Telescope (EIT; Dere et al., 2000) onboard the Solar and Heliospheric Observatory (SOHO) and the Transition Region and Coronal Exploler (TRACE; Handy et al., 1999), AIA uses normal-incidence multilayer mirror coatings to isolate a narrow spectral range $(\approx 10 \AA$ full width at half maximum) for each of its EUV channels; the central wavelengths of the channels are chosen to coincide with strong emission lines formed at different temperatures from $500000 \mathrm{~K}$ to $20000000 \mathrm{~K}$. AIA data consist of images with pixel values $p_{i}(\mathbf{x})$ where the index $i$ refers to one of the ten wavelength channels (nine UV/EUV and one visible light) and $\mathbf{x}$ refers to a location in the field of view. These pixel values are measurements of the solar spectral radiance integrated over the solid angle subtended by the pixel and the wavelength passband of the telescope channel:

$$
p_{i}(\mathbf{x})=\int_{0}^{\infty} R_{i}(\lambda) \mathrm{d} \lambda \int_{\text {pixel } \mathbf{x}} I(\lambda, \theta) \mathrm{d} \theta .
$$

Here $R_{i}$ is the wavelength response function of the $i$-th channel of the telescope, with dimensions of digital number (DN) per unit flux at the aperture. It is possible to recast this measurement equation into an integral over temperature instead of wavelength by using a model of the emissivity of the solar plasma as a function of wavelength and temperature, and folding the emissivity with the wavelength response of the instrument to produce a temperature response function $K(T)$ :

$$
p_{i}(\mathbf{x})=\int_{0}^{\infty} K_{i}(T) \operatorname{DEM}(T, \mathbf{x}) \mathrm{d} T .
$$

Quantitative analysis of AIA data generally consists of using a set of observations to invert (or place constraints on) the spectral distribution of solar emission or the thermal distribution of plasma along the line of sight (the differential emission measure function, $\operatorname{DEM}(T)$ ). In either case, accurate calibration - that is, knowledge of the instrument response as a function of wavelength and temperature - is essential. Relative errors in the calibration of AIA channels can result in much larger distortions in the inferred properties of the emitting region. 
Errors in the absolute calibration can bias the results of an analysis, and make it difficult to take advantage of observations from complementary instruments such as the Hinode/EUV Imaging Spectrometer (EIS; Culhane et al., 2007) and X-Ray Telescope (XRT; Golub et al., 2007 ) to extend the temperature coverage and precision of the AIA observations.

The pre-flight calibration of AIA is described in Boerner et al. (2012), along with a preliminary assessment of the accuracy of that calibration based on early on-orbit data. In this work, we describe a series of experiments to assess and improve the accuracy of the AIA wavelength and temperature response functions by cross-calibration with a number of other instruments. Section 2 describes the testing of the wavelength response with data from SDO/EUV Variability Experiment (EVE) and Hinode/EIS. Section 3 describes the assessment and adjustment of the emissivity function used to generate the temperature response function. In Section 4 we review some of the applications of these results, including tests of differential emission measure inversion using AIA and other instruments.

\section{Wavelength Response}

As noted in Boerner et al. (2012), the wavelength response function of each channel is the product of the effective area $A_{\text {eff }}(\lambda)$ (dimensions of $\mathrm{cm}^{2}$ ) and the gain $G(\lambda)$ (DN/photon). The effective area is the geometrical collecting area of the system, multiplied by the efficiency of each of the components (mirrors, filters, CCD, etc.) as a function of wavelength. The pre-flight calibration relied on component-level measurements of each optical element to determine the effective area and gain. The uncertainty in the wavelength response is thus the stackup of the uncertainties in the calibration of each component, which is approximately $25 \%$. There is additional uncertainty due to changes in the instrument response after the initial measurement due to contamination or other degradation of the instrument. These effects can be significant in the EUV, having resulted in sensitivity losses of a factor of 2 or more on some instruments.

Cross-calibration with other instruments that observe the Sun in the same wavelength channels therefore provides two important capabilites: it enables one to determine the initial calibration accuracy, and it allows for tracking and correction of on-orbit changes in sensitivity. Fortunately, the AIA mission overlaps with the operation of two EUV spectrometers suitable for cross-calibration: SDO/EVE (which measures full-Sun spectral irradiance at high cadence and moderate spectral resolution across the AIA EUV wavelength range), and Hinode/EIS (a slit spectrograph that measures the full range of the AIA $193 \AA$ channel with excellent spatial and spectral resolution).

\subsection{Comparison with SDO/EVE}

The EVE instrument on SDO (Woods et al., 2012) measures the solar spectral irradiance from $60-1050 \AA$ with $\approx 1 \AA$ spectral resolution and a 10 s cadence. While the stated absolute accuracy of EVE's calibration is $25 \%$ (Hock et al., 2012), similar to the expected accuracy of the AIA pre-flight calibration, cross-calibration with EVE provides a number of advantages. EVE is optimized for maintaining accurate absolute calibration. It uses redundant optical elements, proxy models, and comparison with other irradiance monitors to continuously check its measurements, and annual rocket underflights to track degradation.

AIA and EVE measurements are compared as follows: the EVE spectral data (consisting of a solar spectral irradiance $E_{\mathrm{EVE}}(\lambda)$ in units of $\mathrm{W} \mathrm{m}^{-2} \mathrm{~nm}^{-1}$ ) is folded through the AIA wavelength response function $R(\lambda)$ to produce a predicted band irradiance (in $\mathrm{DN} \mathrm{s}^{-1}$ ):

$$
B_{\text {pred }}=\int_{0}^{\infty} E_{\mathrm{EVE}}(\lambda) R(\lambda) \mathrm{d} \lambda .
$$


The predicted band irradiances for each of the AIA EUV channels are computed in the EVE data-processing pipeline for every observation. They are generated using the pre-flight AIA response functions (Boerner et al., 2012) and are included in the Level 2 EVL (extracted lines) data product. Note that the analysis presented here uses Version 2 of the EVE calibration (released in February 2011); it will be updated based on the revisions to EVE's absolute calibration included with the release of Version 3 of the EVE data in March 2013.

The predicted band irradiance is compared with the band irradiance actually observed by AIA $\left(B_{\text {obs }}\right)$. The observed band irradiance is found by summing all the pixels in an AIA Level 1 image (flat-fielded, dark-subtracted, and de-spiked), normalized by exposure time, and adjusted for the distance from AIA to the Sun (since the EVE L2 data are normalized to $1 \mathrm{AU})$. The ratio of the observed AIA count rate to the count rate predicted using the combination of EVE data and the AIA wavelength response function is the EVE normalization factor $F_{\text {norm }}$ :

$$
F_{\text {norm }}=\frac{B_{\text {obs }}}{B_{\text {pred }}} .
$$

EVE observes a larger field of view than AIA, but the amount of irradiance in the AIA bands outside of the AIA field is generally lower than $1 \%$ of the detected irradiance. Because AIA and EVE both operate continuously at a very high cadence, it is possible to compute $F_{\text {norm }}$ for each AIA channel every $12 \mathrm{~s}$ over essentially the full SDO mission.

To track long-term changes in the AIA sensitivity and obtain an overall estimate of the accuracy of the wavelength response function, it is sufficient to sample the normalization factor once per day (averaging $1 \mathrm{~min}$ of AIA and EVE data). Note that EVE only operates the MEGS (Multiple EUV Grating Spectrograph)-B channel (used for the 370-650 A range) for a few hours per day on most days to reduce the dose-dependent degradation of its sensitivity; where possible, we selected the representative minute for each day from the interval when MEGS-B is operational. The results of this long-term comparison using Version 2 of the EVE calibration are shown in Figure 1. A number of features are immediately apparent:

i) For most channels, the ratio is relatively flat or shows a slight degradation in AIA response over time (on the order of $5 \%$ /year or less). The ratios on 1 May 2010, the start of normal science operations, show a DC offset from unity, indicating a discrepancy in the overall normalization of the AIA/EVE calibration. The standard deviation of the offsets in the seven EUV channels is $28 \%$, consistent with our estimate of the accuracy of AIA's preflight calibration.

ii) There are discontinuities in the ratios whenever AIA or EVE performed CCD bakeouts (a list of the bakeouts is in Table 1). EVE bakeouts generally result in a transient uncorrected increase in the EVE signal (within 1-2 weeks after the bakeout, the EVE data have been corrected for the sensitivity changes and the ratios return to their pre-bakeout trend line). AIA bakeouts produce an increase in the ratio, which persists since the AIA data are not corrected based on these measurements. There are occasional discontinuities when the AIA flat-fields are updated (e.g. on 1 January 2012).

iii) There is a long-term drop in $304 \AA$ and $335 \AA$ channel sensitivity. The $304 \AA$ degradation is particularly dramatic, although it appears to have slowed and reversed itself in September 2011. The drop is likely due to the accumulation of volatile contamination on the optics or detector telescopes. Note that the $94 \AA$ channel shares the telescope structure with the $304 \AA$, and the $131 \AA$ channel with the $335 \AA$; however, the typical absorption cross-section of the hydrocarbons associated with contamination is much higher at $\lambda>300 \AA$ than at $\lambda<150$, so a thin layer of contamination might easily attenuate the $304 \AA$ by a factor of two without having a noticeable effect on the $94 \AA$ (Boerner et al., 2012). 


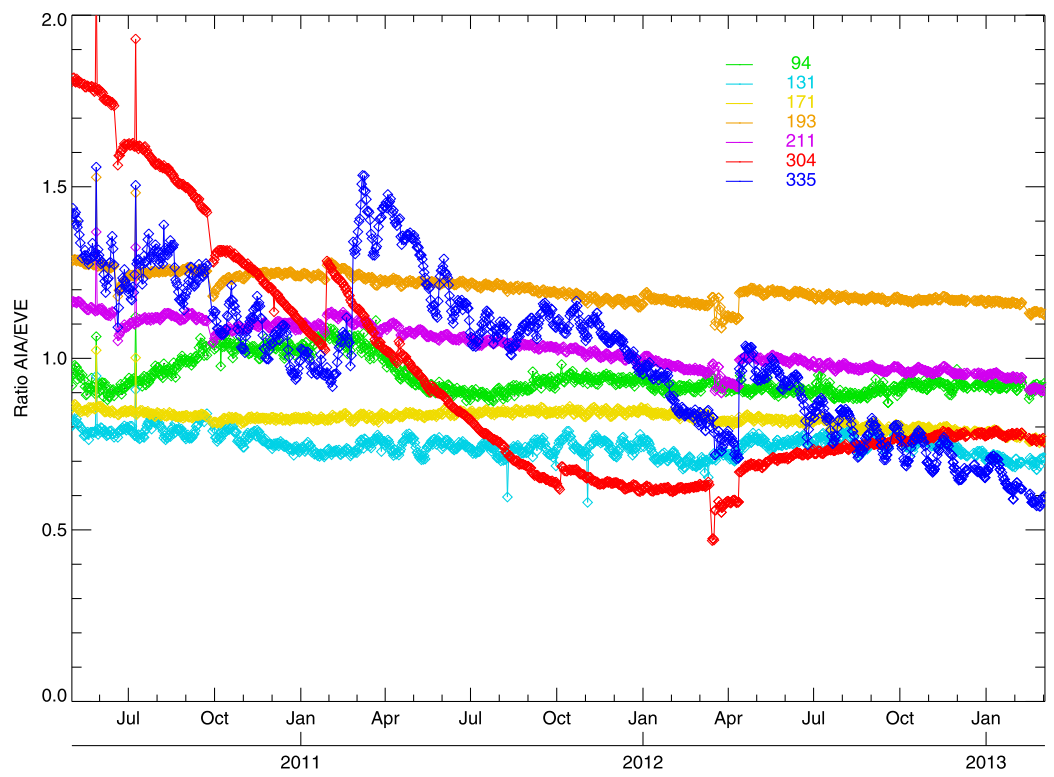

Figure 1 The ratio of the total irradiance observed in each AIA EUV bandpass to that predicted by folding EVE spectra through the AIA pre-flight wavelength response functions. If we assume the EVE data are perfect, this ratio can be used as a correction factor for the AIA wavelength response.

Table 1 History of bakeouts performed on AIA Telescope Assembly (ATA) and EVE/MEGS. ${ }^{\mathrm{a}}$ Heated entire telescope, not just
CCD.

\begin{tabular}{llcl}
\hline Date & $\begin{array}{l}\text { Instrument } \\
\text { affected }\end{array}$ & $\begin{array}{l}\text { Approximate } \\
\text { duration }[\mathrm{h}]\end{array}$ & $\begin{array}{l}\text { Temperature } \\
{\left[{ }^{\circ} \mathrm{C}\right]}\end{array}$ \\
\hline 18-Jun-2010 & EVE/MEGS & 240 & \\
24-Sep-2010 & EVE/MEGS & 240 & \\
28-Jan-2011 & ATA2, 3, 4 & 2 & 10 \\
25-Feb-2011 & ATA1 & 2 & 10 \\
14-Apr-2011 & ATA4 & 24 & 10 \\
19-May-2011 & ATA4 & 8 & 20 \\
4-Oct-2011 & ATA4 & 36 & $20^{\mathrm{a}}$ \\
12-Mar-2012 & EVE/MEGS & 72 & 10 \\
12-Apr-2012 & ATA1, 2, 3, 4 & 2 & \\
\hline
\end{tabular}

iv) The $335 \AA$ ratio shows much greater variation on the timescale of the solar rotation $(10 \%)$ than any of the other channels (typically smaller than $1 \%$ ). This may indicate that the assumed shape of the $335 \AA$ wavelength response function is incorrect, causing the ratio to vary depending on the spectral distribution of the solar irradiance. However, efforts to flatten out the ratio by iteratively adjusting the wavelength response function have not enabled us to produce a realistic alternate response function that reduces the variation in the ratio while remaining compatible with the uncertainties in the instrument calibration. It is also possible that signal from higher orders in the EVE spectrum around $335 \AA$ may cause these ripples (in which case the shape of the wavelength response function may be correct). 
v) The $94 \AA$ channel shows some modulation on the timescale of one year. This is attributable to the change in the $94 \AA$ flatfield due to burn-in by the $304 \AA$ image on their shared detector (Shine et al., 2010), an effect that was not corrected for until January 2012. The CCD area corresponding to the solar disk image at $304 \AA$ has a slightly lower sensitivity at $94 \AA$; thus, when SDO is at aphelion and the solar image is smallest, more of the $94 \AA$ flux (which is preferentially distributed at and above the solar limb) falls on the affected area of the detector, and thus the observed $94 \AA$ irradiance is lowest in July.

Some of the offset from unity and the long-term trends noted in Figure 1 may be attributable to errors in EVE's calibration, and not in AIA's. However, since EVE is generally expected to have a better absolute calibration and has a much better mechanism for tracking on-orbit degradation (through sounding rocket underflights), we might improve AIA's calibration by adjusting the wavelength response functions by $F_{\text {norm }}$ so that the EVE-predicted band irradiances match the observations. The normalization factor is a function of time; we can approximate it as a series of polynomials for each channel and each time interval $j$ between bakeouts of that channel:

$$
F_{\text {norm }}^{\prime}(t)=\sum_{i=0}^{n} p_{i j}\left(t-t_{j}\right)^{i} .
$$

This is similar to the approach used in Hock and Eparvier (2008) for cross-calibration of EIT and TIMED/SEE. The time-dependent approximated normalization factor was used to compute corrected predicted band irradiances $B_{\text {corr }}(t)=F_{\text {norm }}^{\prime}(t) B_{\text {pred }}(t)$. The accuracy of the correction was determined by examining the residual ratios of this fit, $B_{\text {obs }} / B_{\text {corr }}$ (see Figure 2). We found that the residual deviations from unity for all EUV channels other than $335 \AA$ are smaller than $4 \%$ RMS using a polynomial of order $n=0$ or 1 . The polynomial coefficients $p_{i j}$ and epoch start times $t_{j}$ used to compute $F_{\text {norm }}^{\prime}(t)$ are included in the SolarSoftware (SSW; Freeland and Handy, 1998 ) routine aia_get_response, which was used to access the wavelength and temperature response functions.

The spectral resolution of EVE, while considerably higher than that of the AIA channels, may not be high enough to avoid introducing some bias into this determination of the correction factor. To assess this possibility, we simulated a solar spectrum at very high resolution $(0.05 \AA)$ using the CHIANTI atomic database (Landi et al., 2012). We first folded this spectrum through the AIA wavelength response functions to produce a predicted count rate, then blurred the spectrum with a Gaussian width of $0.47 \AA$ and downsampled it to 0.16 $\AA$ spectral bins (which produces a good empirical match with the appearance of the lines in the EVE Level 2 spectra around $200 \AA$ ). We compared the count rate predicted using the blurred spectrum with that predicted by the full-resolution spectrum (Figure 3). In most cases, the differences were smaller than $1 \%$; however, for the $171 \AA$ channel (where there is a strong solar emission line from Fe IX next to the sharp Al L-edge in the response function) the slight blurring was enough to reduce the predicted count rate by approximately $10 \%$. For the $94 \AA$ channel (which is very narrow), the effect was an underprediction of $30-40 \%$ (depending on the relative strength of the Fe XVIII line). This implies that while the agreement between AIA and EVE appears to be quite good in the $94 \AA$ channel, it is possible that the assumed effective area for this channel is too high (the calibration error may be compensating for the effect of EVE's spectral resolution).

Note that a similar effect applies when one attempts to fold the EVE EVS Level 2 spectral data through the response functions to reproduce the band irradiances reported in the EVL line products: the Level 2 spectra are rebinned to a slightly coarser grid than the unpublished Level 1 data used to calculate the EVL band irradiances (Woods et al., 2012), and thus give 

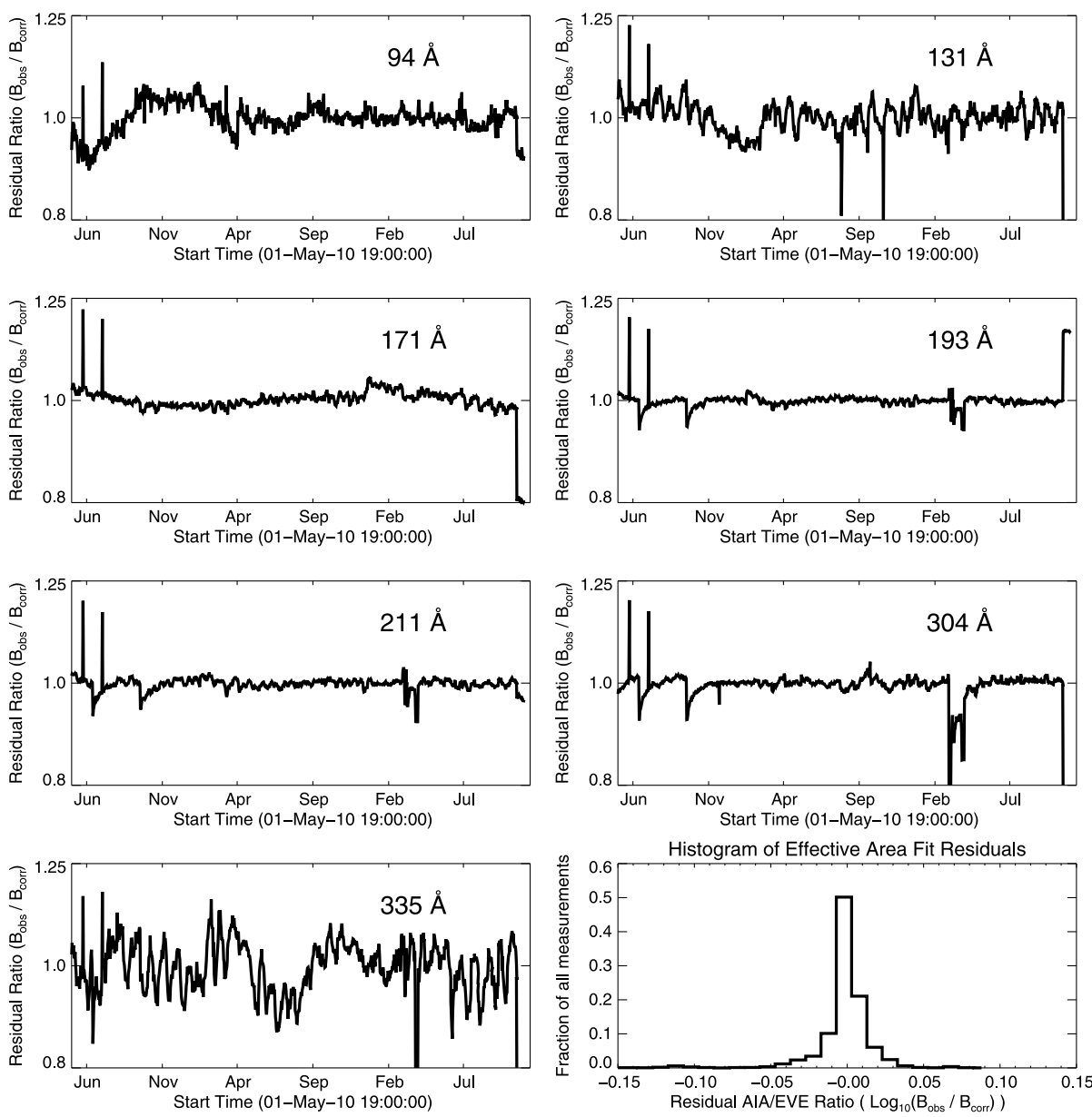

Figure 2 The residual ratios left from fitting the AIA/EVE ratio in the intervals between AIA bakeouts with a flat or linear function of time. At bottom right is a histogram of the residuals from all EUV channels, showing that the vast majority of daily samples are within $2 \%$ of the fit value.

an answer that is up to $20 \%$ lower for the 94 and $171 \AA$ channels. For this reason, we used the EVL data for all comparisons.

\subsection{Comparison with SORCE/SOLSTICE}

EVE does not cover the wavelength range of the AIA UV channels (1500-1800 ̊); however, SORCE/SOLSTICE (McClintock, Rottman, and Woods, 2005) measurements are available in this range. The approach described above can be used to fold SORCE/SOLSTICE data through the AIA UV channel response functions and compare the predicted and observed band irradiances for the 1600 and $1700 \AA$ channels. While the spectral resolution of SOLSTICE is roughly an order of magnitude lower than that of EVE, the AIA UV passbands are comparably broader than the EUV bands, and the solar spectrum in this range is less dominated by sharp lines, so the blurring of the spectrum by the instrumental response of SOLSTICE has a negligible impact on the predicted count rates. The results are shown 
Figure 3 To estimate the impact of EVE's spectral resolution on the comparison with AIA observations, a simulated high-resolution spectrum (black) is blurred and downsampled to match the appearance of EVE lines (blue). The blurred spectrum is folded through the AIA wavelength response (red), and the resulting count rate is compared with that predicted using the unblurred spectrum. The top and the bottom panels are for the 94 and $171 \AA$ channels, respectively.
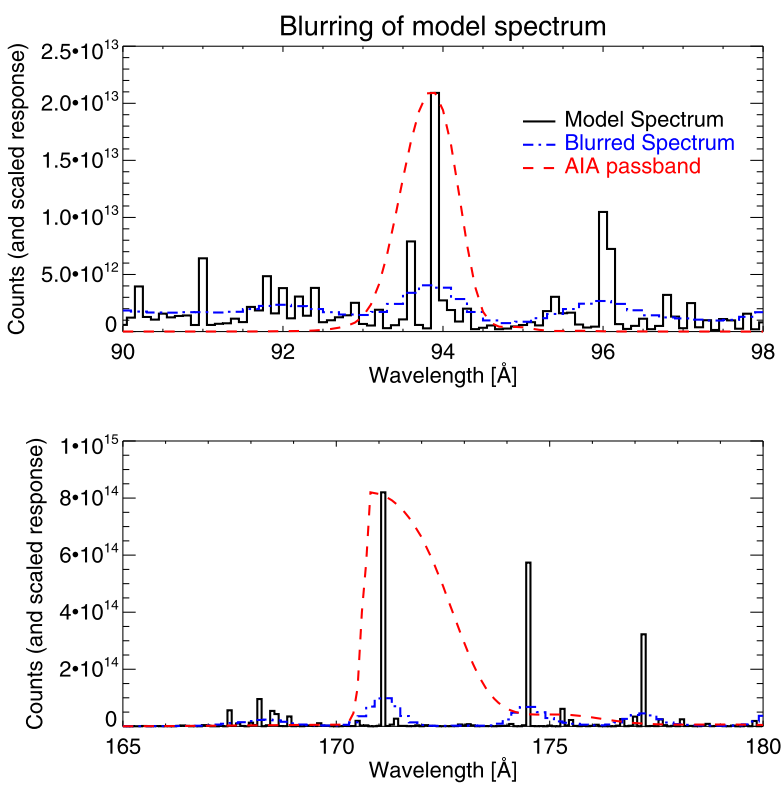

Figure 4 The ratio of band irradiances measured in the AIA UV channels to those predicted using data from

SORCE/SOLSTICE. As noted in Boerner et al. (2012), the absolute accuracy of the AIA UV channel calibration is poorer than for the EUV channels (a factor of 2 instead of $25 \%$ ). However, the trend plot shows only weak shortand long-term variation. (The step on 1 January 2012 is due to a change in the normalization of the AIA flatfield.)

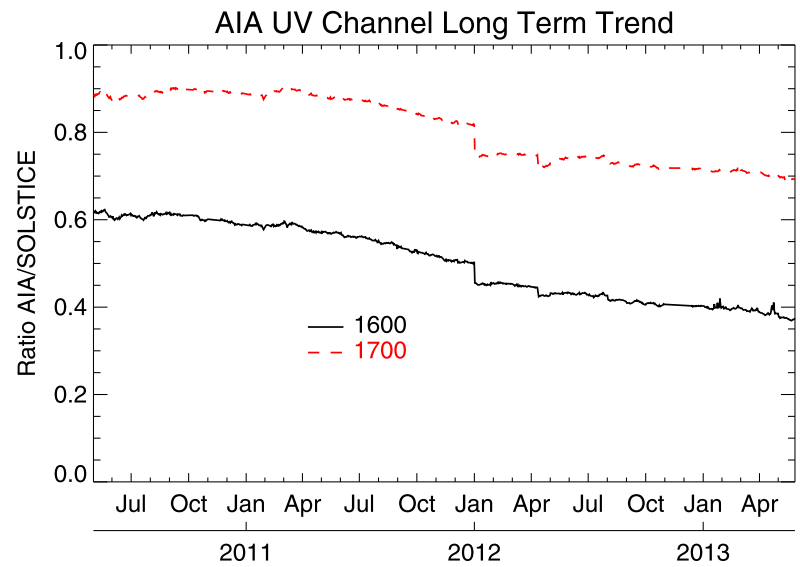

in Figure 4. Again, low-order polynomials produce excellent fits to the observed trends with residuals $<4 \%$. These fits are available through SSW.

\subsection{Comparison with Hinode/EIS}

The EIS instrument on Hinode (Culhane et al., 2007) is a slit spectrograph that operates in two EUV wavelength bands; the shorter band (170-210 $\AA$ ) completely overlaps the AIA $193 \AA$ channel. EIS offers excellent spectral resolution (approximately $50 \mathrm{~m} \AA$ ), with a spatial resolution of 2 arcsec; it can be rastered to produce images with a field of view of $6 \times$ 8.5 arcmin. While cross-calibration between EIS and EVE is difficult because of their discrepant fields of view, EIS has been cross-calibrated by the EUNIS sounding rocket (Wang et al., 2011) and with SOHO/SUMER (Landi and Young, 2010). 

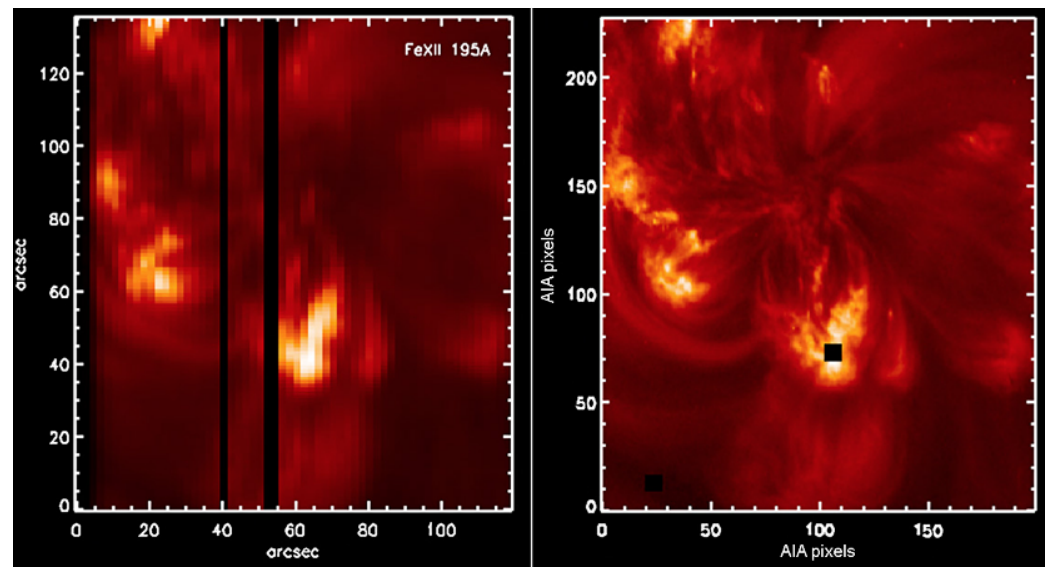

Figure 5 (Left) A simulated AIA $193 \AA$ raster constructed by multiplying a 3D EIS spectral data cube with the AIA $193 \AA$ response function and integrating over wavelength. (Right) AIA $193 \AA$ observations of the same region; this is a pseudo-raster, as each pixel is chosen from an image taken at the same time as the corresponding EIS integration. The circled areas indicate the location of the bright "moss" and dim "quiet-Sun" sub-regions selected for detailed comparison.

Table 2 EIS vs AIA $193 \AA$ channel.

\begin{tabular}{ll}
\hline Feature & AIA observed/EIS predicted \\
\hline Moss & 1.03 \\
Quiet Sun & 0.98 \\
Full FOV & 1.15
\end{tabular}

To compare AIA and EIS observations, it is necessary to ensure that they are observing the same field. The EIS spectral data cube from a slit raster $I(\mathbf{x}, \lambda)$ was multiplied by the AIA $193 \AA$ response function $R(\lambda)$ and integrated over wavelength to produce a set of predicted $193 \AA$ pixel intensities $p_{\text {pred }}(\mathbf{x})$. Then AIA $193 \AA$ images were used to build a "simulated raster" $p_{\text {obs }}(\mathbf{x})$ such that each pixel in the result was chosen from an image taken at the same time as the corresponding EIS slit integration. The AIA/EIS normalization factor is then the ratio of $p_{\mathrm{obs}} / p_{\text {pred }}$ for all points in the image.

This technique was applied to an EIS raster taken in October 2010 (see Figure 5). The field of view contained a small active region, including some moss and some patches of quiet Sun. While the pixel-to-pixel variations in the AIA/EIS normalization factor could be substantial because of the difficulty in exactly co-aligning each pixel in space and time, the average over regions as small as $20 \times 20$ arcsec showed good agreement to within $15 \%$ for the moss, quiet Sun, and the full field of view (see Table 2).

\section{Temperature Response}

The temperature response function, $K(T)$, of an EUV narrowband imager is calculated from the wavelength response function and the plasma emissivity $G$ :

$$
K_{i}(T)=\int_{0}^{\infty} G(\lambda, T) R_{i}(\lambda) \mathrm{d} \lambda .
$$


The emissivity is a description of the plasma and atomic physics that govern how material at a given temperature emits radiation. It includes empirically derived values of the abundance of the various elements in the solar atmosphere, the ionization equilibrium of the ionic species of each element as a function of temperature, and the oscillator strengths of all the known emission lines of each ion (as well as a model of the continuum emission). This information is contained in the CHIANTI database, which represents a compendium of measurements and theoretical calculations of plasma properties.

Compiling the emissivity database and code is a challenging, ongoing research program, so the uncertainties associated with the emissivity are not negligible. For many of the emission lines targeted by AIA, the CHIANTI database is quite accurate; in particular, at wavelengths above the Al-L edge at $171 \AA$, there have been numerous measurements of solar and stellar intensity, which have been used to refine the emissivity models (the same is true, to some extent, for the soft X-ray region between 6 and $50 \AA$ ). However, prior to the launch of SDO, there had been very few measurements in the 50-150 ̊ range, and as a result the emissivity in this range was only poorly characterized.

\subsection{Benchmarking CHIANTI}

Based on the observations of the 50-150 A range with EVE and the 94 and $131 \AA$ channels on AIA, it is clear that there are significant deficiencies in the spectral models in this wavelength range. Figure 6 shows an observed irradiance spectrum of the non-flaring Sun from EVE (black), along with a best-fit model spectrum generated using CHIANTI Version 7.0 (red) and 7.1 (green). The model shows excellent agreement with the many strong lines between $170-350 \AA$ (with the well-known exception of the $304 \AA$ He II line), implying that the assumptions about the thermodynamic state of the plasma are good. But between 50 and $\approx 150 \AA$ the model fails to reproduce the majority of the emission lines, and underpredicts the observed intensity by factors of $2-6$. CHIANTI 7.1 clearly represents a substantial improvement, but there is still a significant amount of emission that is not accounted for. The missing flux is most significant in the quiet Sun; during flares, the emission in this wavelength range is dominated by a handful of strong lines (such as Fe XVIII $94 \AA$ and Fe XXI $128 \AA$ imaged by AIA) that are well-reproduced by CHIANTI. However, the underestimate of the intensity from quiet Sun plasma can lead to false conclusions about the presence of hot plasma.

This effect has been independently discovered by a number of authors (e.g. Aschwanden and Boerner, 2011; Teriaca, Warren, and Curdt, 2012). To prove that this discrepancy is not a result of a calibration error in EVE, Testa, Drake, and Landi (2012) also examined spectra of Procyon taken by Chandra's LETG. Again, they found that the CHIANTI model (which agrees well with the observed line intensities at more well-studied wavelengths) simply does not contain any information for many of the lines in this spectral range.

\subsection{Empirical Correction to AIA Temperature Response}

Work is in progress to update CHIANTI to include these missing lines (see, e.g., Del Zanna et al., 2012); the release of Version 7.1 represents a major step. However, in the mean time, it is possible to make an empirical correction to the AIA temperature response functions themselves to attempt to account for the missing emission. This is done using the dataset of coordinated observations with AIA and EVE during a 1-h window around the X2 flare of 15 February 2011, and in samples of the irradiance taken daily throughout the SDO mission. (The flare spectra have a pre-flare baseline subtracted to isolate the dynamic hot component 

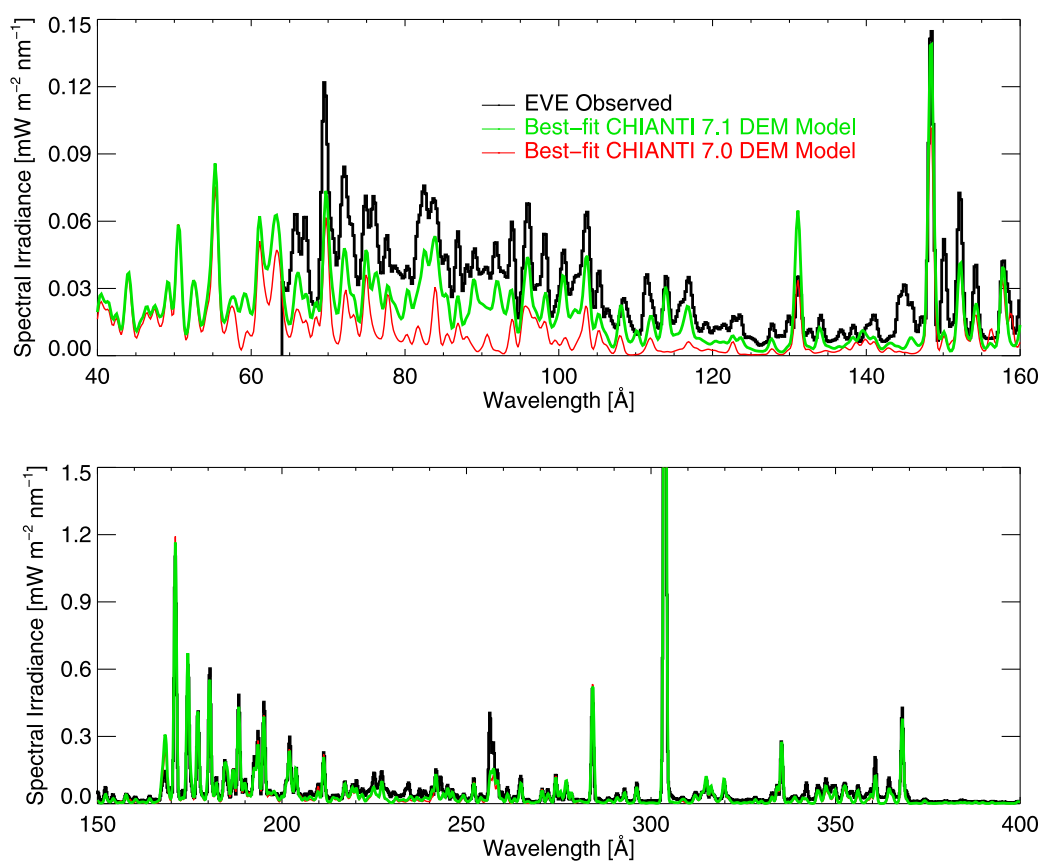

Figure 6 An observed irradiance spectrum from SDO/EVE, compared with the best-fit spectrum using a DEM model and the CHIANTI database. The model accurately matches the observations in the $170-350 \AA$ range, but significantly underestimates the emission from $60-170 \AA$.

of the emission.) The EVE data are used to constrain a model of the DEM as follows. The quiet-Sun DEM derived by Dere et al. (1997) from the observations of Vernazza and Reeves (1978) is used as an initial guess, and parameterized as a cubic spline in $\log _{10}(T)$ using $4-6$ spline knots. The DEM is combined with the emissivity function derived from CHIANTI to generate a synthetic spectrum,

$$
I(\lambda)=\int_{T_{\min }}^{T_{\max }} G(\lambda, T) \operatorname{DEM}(T) \mathrm{d} T .
$$

The synthetic spectrum is blurred and resampled to EVE resolution as described in Section 2.1, and the result is compared with the observed EVE Level 2 Version 2 spectrum in a set of windows $2 \AA$ wide centered on a set of strong emission lines in the spectral range where the CHIANTI model is known to be reasonably complete, and a $\chi^{2}$-value is calculated by summing the squared differences of all EVE spectral bins in the selected windows. (Using windows instead of attempting to extract line intensities from the EVE measurements gives results that are more robust to blending that might result from EVE's moderate spectral resolution.) Note that the spectral windows around certain lines associated with high-temperature emission found in flares, including the Fe XVIII $94 \AA$ line and the Fe XXI $128 \AA$ Aine imaged by AIA, are treated as upper limits and only factor into the $\chi^{2}$-value when the predicted intensity exceeded the observed intensity; this allowed us to use these lines to constrain the hot end of the DEM during flares (since the CHIANTI data are fairly accurate for these hot lines), without being misled by the deficiencies in the CHIANTI model of the adjacent cooler lines. 
The DEM spline knots were then adjusted iteratively using the Levenberg-Marquardt algorithm (the mpfit routine in IDL) to minimize the $\chi^{2}$. The DEM functions derived using this approach generally fit the EVE observations in the selected windows to better than $25 \%$ (see Figure 7), so they can be considered a reasonably good representation of the thermal state of the corona. The comparison between the observed and best-fit synthetic spectra over the full EVE spectral range for both the daily sampled spectra and the $\mathrm{X} 2$ flare spectra (with the pre-flare spectrum subtracted) can be seen in movies posted at http://www.Imsal.com/ boerner/crosscal/. A number of characteristics of these movies are worth noting:

i) For the flare spectrum, the strong lines in the 94 and $131 \AA$ bands are fit quite well (the cooling of the flare from Fe XXI to Fe XVIII is apparent). The 193 and $335 \AA$ bands also match reasonably well.

ii) However, the 171 and $211 \AA$ channels do not match the preflare-subtracted observations. This is most likely because these channels do not have a significant contribution from hot lines, so the enhancement to their irradiance during the flare is negligible compared to fluctuations (or even dimmings; see, e.g., Woods et al., 2011) in the global 1-2 MK corona; therefore, subtracting a static pre-flare background leaves only noise in these bands.

iii) The daily samples (which typically resemble an average quiet-Sun DEM) generally agree very well in the range from $170-200 \AA$ including the lines not used in the fit.

iv) There are some spectral ranges that are not well fit for the daily samples, including $200-250 \AA$ and $320-360 \AA$. This is probably because the DEM is only poorly constrained below about $\log _{10}(T)=5.6$; however, this temperature range is not of primary significance for AIA.

v) Of course, the quiet-Sun DEMs consistently underestimate the observations in the region from $60-150 \AA$, as expected (see Section 3.1).

After determining DEM functions that accurately characterize the corona, we adjusted the AIA temperature response functions so that the count rate predicted by folding these DEMs through the response functions using Equation (2) matched the observed AIA band irradiance.

The adjustment of the temperature response functions is a two-step process. Because we believe that CHIANTI accurately predicts the intensity of the hot lines that dominate during flares, any discrepancy between observed and DEM-predicted count rate using backgroundsubtracted flare observations can be attributed to a normalization error in the temperature response function, and we can simply determine a scale factor $a_{0}$ that optimizes the agreement:

$$
K_{\text {scale }}(T)=a_{0} K_{\text {orig }}(T) .
$$

The results for the 94 and $131 \AA$ channels are shown in Figure 8. Note that the 12-min period around the peak of the flare is omitted from the fit because substantial saturation in the AIA image reduces the reliability of the AIA irradiance measurements. The band irradiance predicted using the scaled temperature response functions matches the observations very closely. The best-fit scale factors are 0.62 for the 94 channel (i.e. the count rates are only $62 \%$ of what would be predicted using the nominal temperature response function and the best-fit flare DEMs), and 0.63 for the $131 \AA$ A channel.

We then compare this scale factor derived in temperature space with the $F_{\text {norm }}$ derived in wavelength space using the same dataset (by folding the preflare-subtracted EVE spectral irradiance through the wavelength response function, as in Section 2.1). For the $131 \AA$ channel, the wavelength comparison suggests a correction factor of 0.64 , which is quite close to 

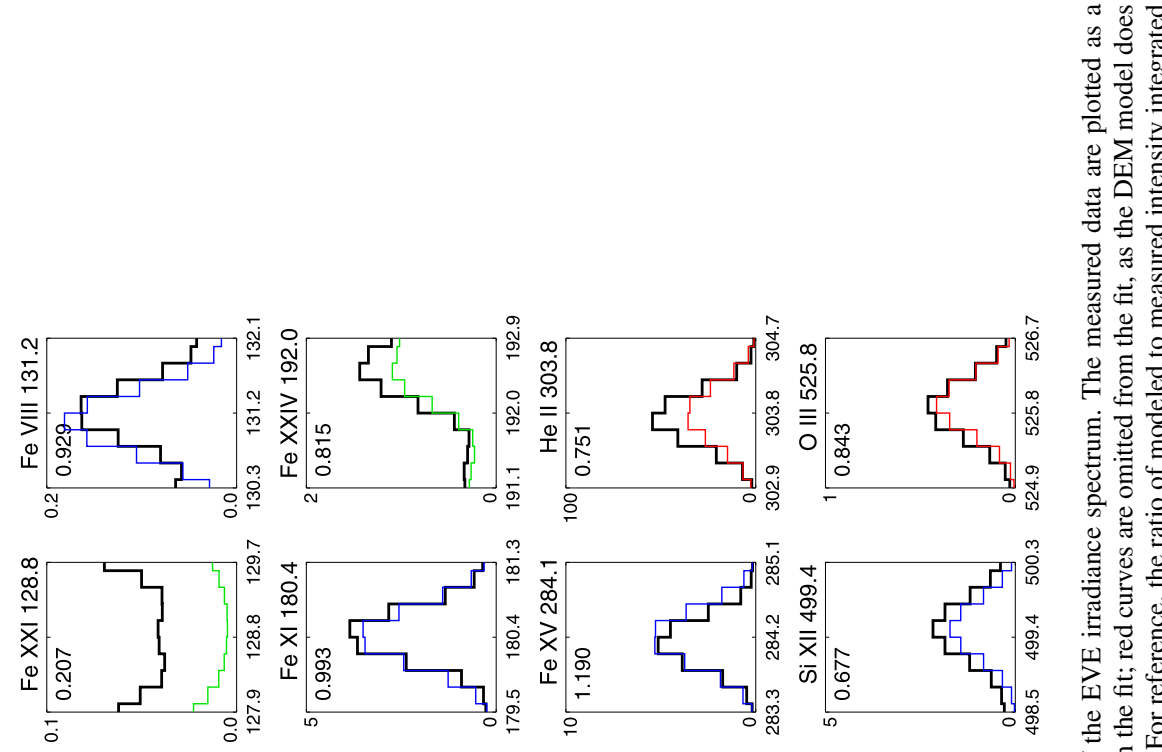

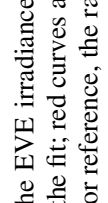

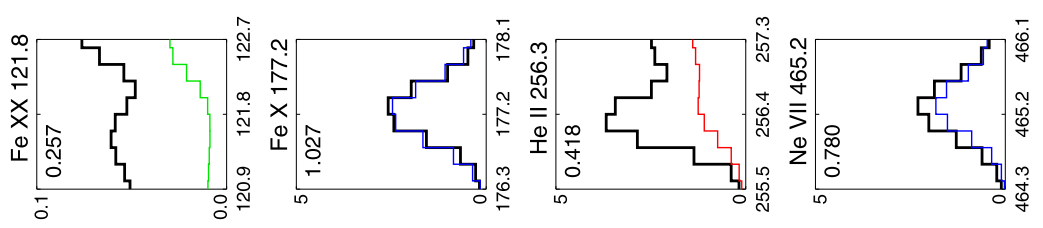

$4 . \Xi$

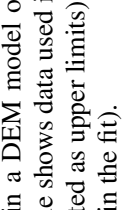

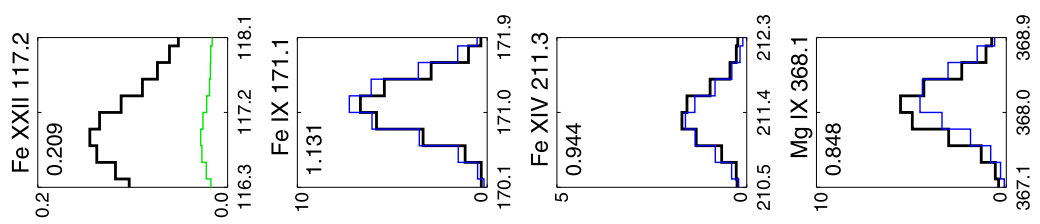

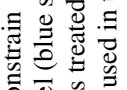

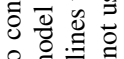

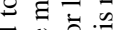

D.

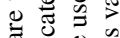

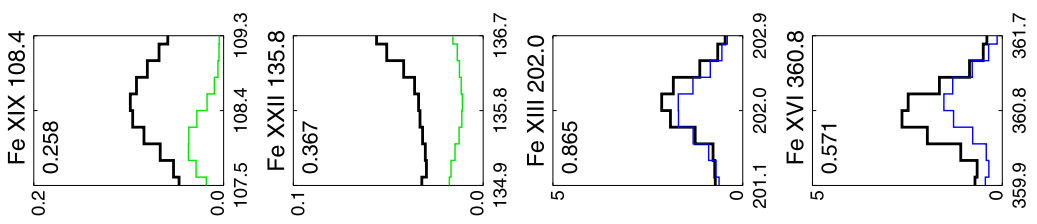

น

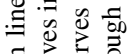

.

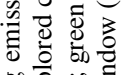

on 0

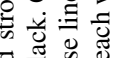

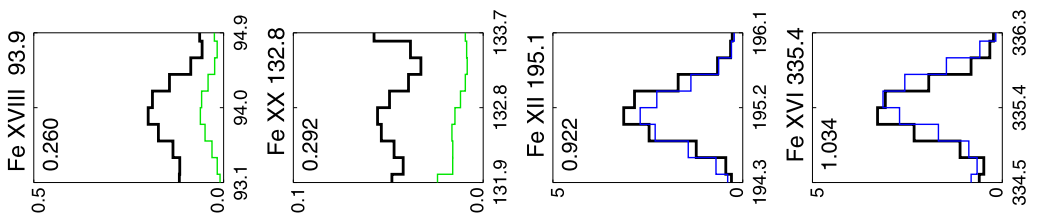

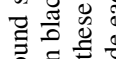

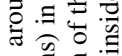

है ฮี ซี

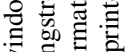

콩

क

प릉

远导

$\varangle \frac{\pi}{3} \frac{\pi}{0}$

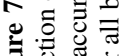

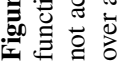


Figure 8 The DEMs derived from the flare observations are used to normalize the temperature response functions. The irradiance predicted using the DEMs and the temperature response functions with the best-fit normalization constant closely matches the observed irradiance during the cooling phase of the flare.
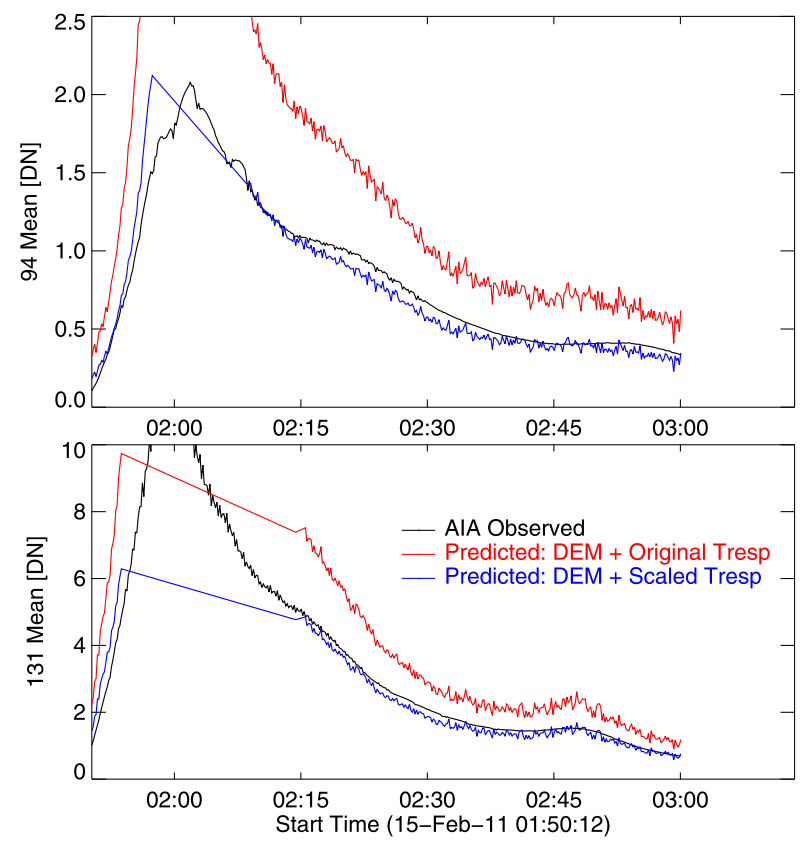

what we find in temperature space. However, we note that the wavelength space comparison gives a correction factor of 0.81 when we look at the spectrum before and after the flare, without subtracting the baseline. We interpret this to mean that the effective area of the $131 \AA$ channel needs to be scaled by 0.64 at the wavelength of the Fe XXI flare line, but only by 0.81 at the wavelength of the Fe VIII line that dominates in non-flaring conditions. Instead of attempting to adjust the shape of the wavelength response function, we adjusted the entire response function by 0.81 to agree with the wavelength cross-calibration during non-flaring times, and then applied an additional scale factor of 0.79 to the portion of the temperature response function above 6.7 in $\log _{10}(T)$.

For the 94 channel, the correction derived in wavelength space is close to 1.0 than to the 0.62 derived in temperature space. Most of the discrepancy can be attributed to the wavelength resolution effect noted above; if we take the synthetic spectrum predicted by the best-fit flare DEMs and blur it to EVE's spectral resolution, the predicted count rates in the $94 \AA$ channel are approximately $30 \%$ lower than the predictions obtained with the unblurred spectrum. The remaining $8 \%$ discrepancy may be attributable to errors in the DEM fit. However, note that the adjustment to the high-temperature component of the 94 channel temperature response derived from this comparison is most likely more accurate than the adjustment implied by, and could not be obtained directly from, folding the EVE observations through the wavelength response function. Therefore, we scaled the entire 94 Å channel response down by 0.7 .

After fixing the normalization of the responses such that they agree excellently with EVE spectra and with EVE-constrained DEMs during flares, the next step is to add come contribution to the lower-temperature portion of the functions so that the daily sample DEMs accurately predict the observations,

$$
K_{\text {fit }}(T)=K_{\text {scale }}(T)+\sum_{n=1}^{2} a_{n} G_{n}(T) .
$$




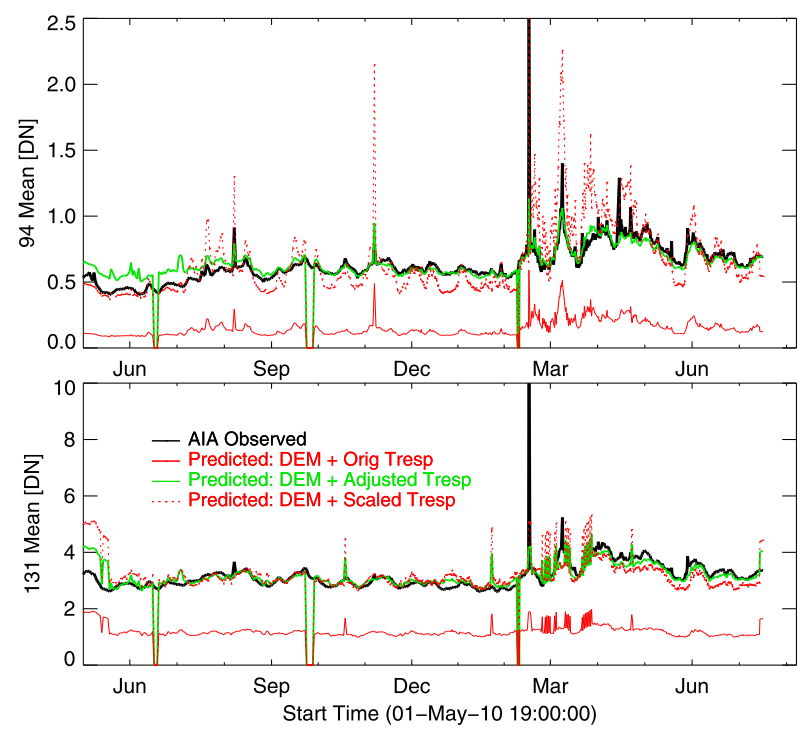

Figure 9 Daily samples of the EUV irradiance taken over a broad range of solar conditions were used to constrain the shape of the cool end of the temperature response functions. The original temperature response functions (red) underestimate the observations (black) by factors of 2-4. Contributions from Fe VIII-XII were added until the agreement between the observed band irradiance (black) and the count rate predicted using the best-fit DEM and the modified temperature response (green) matched the magnitude and the variation of the observations. Simply scaling up the cool portion of the original temperature response function by a best-fit factor (red dashed line) matches the average value of the signal, but not the details of its variation.

The shapes of the contribution functions $G_{n}(T)$ are chosen based on estimates of the temperature characteristics of the emission missing from each bandpass, derived either from surveys of the atomic databases (see, e.g., Del Zanna (2012), who noted that there are probably strong Fe IX lines missing from the $94 \AA$ channel) or from comparing the morphology of structures seen in the images to images from lines at well-known temperature (Warren, O'Brien, and Sheeley, 2011). Note that in the quiet Sun, the $94 \AA$ images most closely resemble EIS and AIA Fe XII images. The $a_{n}$ coefficients are then found by minimizing the $\chi^{2}$. For the $94 \AA$ channel, we chose $G_{1}(T)$ to be the temperature distribution of the Fe IX line at $171 \AA$ and $G_{2}(T)$ to be the shape of the Fe XII $195 \AA$ line. For the $131 \AA$ channel, $G_{1}(T)$ was based on the $180 \AA$ Fe XI line, and $G_{2}(T)$ was the shape of the Fe VIII line already in the $131 \AA$ band. Alternate parameterizations were tried, with $n=1$ to $n=3$ and different temperature lines added to each band. The results are not very sensitive to the exact details of the added contribution; for example, agreement between predicted and observed counts in the $131 \AA$ channel would not be very different if we chose to add an Fe X-like component instead of an Fe XI-like component, and the relative balance of Fe IX and Fe XII added to the $94 \AA$ channel is poorly constrained. However, the basic shape of the corrections is well-motivated and provides very good agreement with observations.

This agreement is shown in Figure 9. The observed band irradiances are plotted in black, and the predictions given the best-fit DEMs and the original temperature response functions $K_{\text {orig }}(T)$ are shown in red, while the predictions obtained with the best-fit response functions $K_{\text {fit }}(T)$ are shown in green. Note that the predictions obtained by simply scaling up the cool end of the temperature response function (as was done in Aschwanden and Boerner, 2011), plotted with dotted red lines, improve the agreement substantially, but clearly do not match 

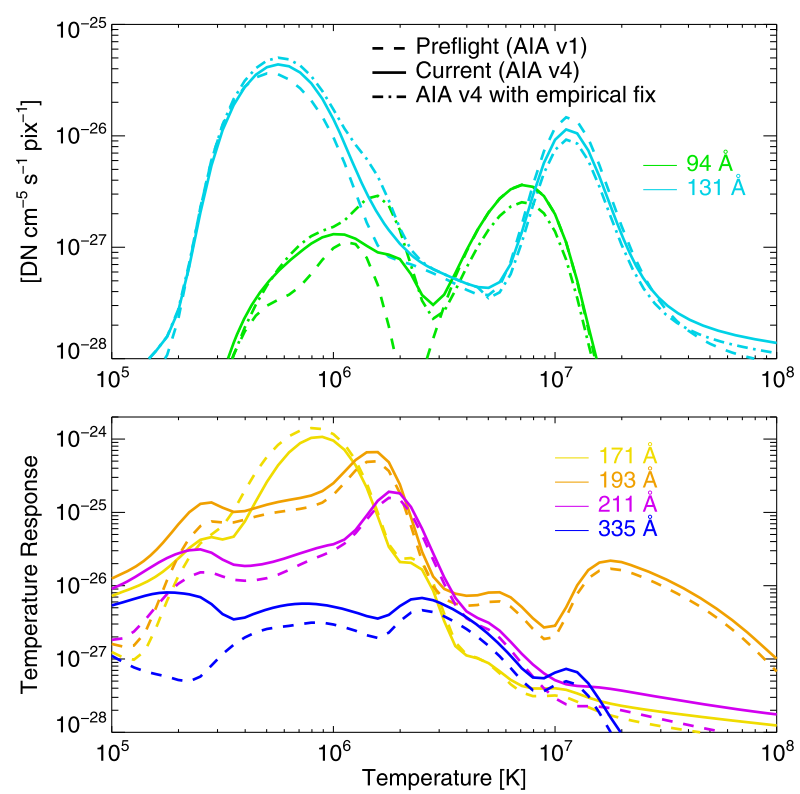

Figure 10 The temperature response functions for the AIA EUV channels with the corrections discussed here applied. The pre-flight calculation (using ground calibration of the effective area combined with atomic data from CHIANTI Version 6.0.1) is shown with a dashed line (this is Version 1 of the AIA calibration). The updated temperature response calculated by cross-calibration of the wavelength response function with EVE combined with atomic data from CHIANTI Version 7.1 is shown with the solid lines. In the top panel, the empirical correction to the 131 and $94 \AA$ channels is also shown with a dash-dotted line. For both channels, the high-temperature peak is slightly reduced, and there is significant additional contribution from material around $1 \mathrm{MK}$.

Table 3 AIA calibration version history.

\begin{tabular}{lllllll}
\hline $\begin{array}{l}\text { AIA calibration } \\
\text { version }\end{array}$ & $\begin{array}{l}\text { Release } \\
\text { date }\end{array}$ & $\begin{array}{l}\text { CHIANTI } \\
\text { version }\end{array}$ & \multicolumn{4}{l}{ Approx. scale of empirical fix } \\
\cline { 5 - 7 } & Hot 94 & Cool 94 & Hot 131 & Cool 131 \\
\hline 1 & Aug 2010 & 6.0 .1 & - & - & - & - \\
2 & Feb 2012 & 7.0 & 0.55 & 4.0 & 0.85 & 2.0 \\
3 & Sep 2012 & 7.0 & 0.55 & 4.0 & 0.85 & 2.0 \\
4 & Feb 2013 & 7.1 & 0.70 & 2.0 & 0.79 & 1.0 \\
\hline
\end{tabular}

the detailed behavior of the observations as well as the best-fit modifications, especially in the $94 \AA$ channel. The best-fit response functions are shown in Figure 10.

As noted in Section 3.2, Version 7.1 of CHIANTI (released in October 2012) added a large number of emission lines in the 50-160 $\AA$ range and thus reduced the need for and the impact of the empirical correction to the AIA temperature response. The AIA response functions were updated to Version 4 to incorporate these new emission lines, with the empirical correction (accessible with the chiantifix keyword to the aia_get_response function) retuned appropriately. The history of the AIA calibration versions is summarized in Table 3. 

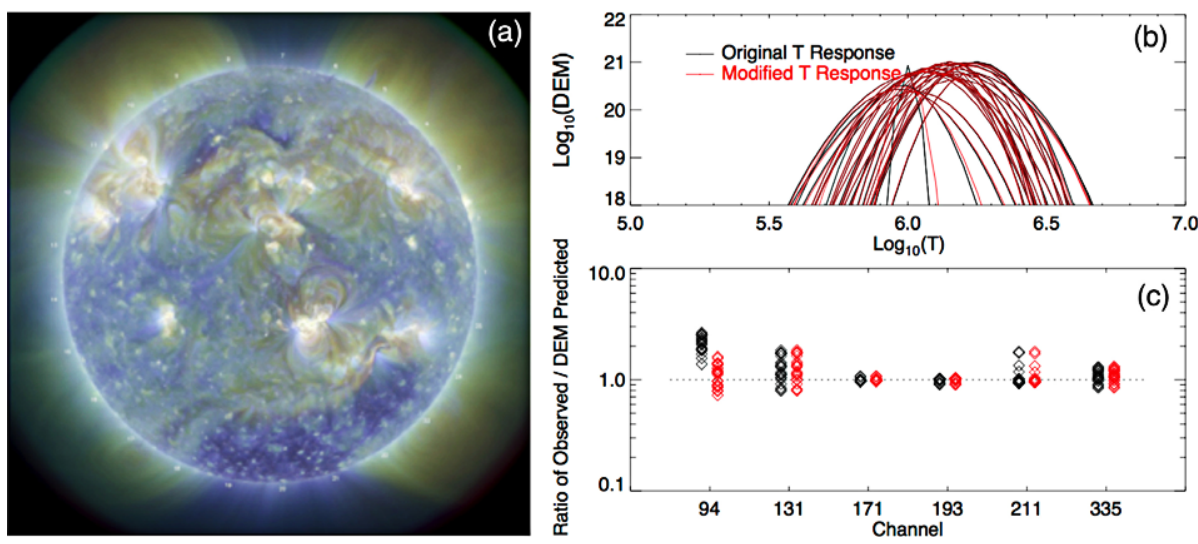

Figure 11 The region above the solar limb was divided into 25 sectors (a). The best-fit Gaussian DEMs are shown in (b), along with the ratio of the observed count rate in each channel to that predicted by the DEM (c). The modified temperature response functions produce much better agreement in the 94 and 131 channels.

\section{Implications for Thermal Analysis}

To validate these results on a separate set of observations and to characterize their effect on the conclusions obtained from thermal analysis with AIA, we carried out a series of inversions using both the original and the modified temperature response functions.

\subsection{DEMs with AIA Alone}

For the first of these, we used only AIA data. The six Fe channels of AIA can provide reliable temperature constraints with moderate resolution $\left(0.3\right.$ in $\left.\log _{10}(T)\right)$ for optically thin plasma in the range of 0.7-3 MK (Guennou et al., 2012). Averaging over large regions of the corona above the limb during non-flaring conditions therefore provides an effective benchmark for DEM inversions. We divided the off-limb corona from the period prior to the X2.2 flare on 15 February 2011 into 25 sectors of equal size and integrated the signal into the six Fe channels from each sector.

For each sector, a DEM inversion was performed using a single-Gaussian function of temperature, with both the original (black in Figure 11) and modified (red in Figure 11) temperature response functions. Because the 171, 193, and $211 \AA$ channels are an order of magnitude more sensitive to plasma at the temperature of the quiescent corona, their signals dominate the fit. The recovered DEM functions show only minor differences when the modified 94 and $131 \AA$ A responses are used, generally producing slightly narrower Gaussians. However, the modified responses dramatically improve the agreement with the 94 and 131 $\AA$ observations. With the original response functions, the Gaussian DEMs underpredict the flux in both channels by the same factor of 2-4 noted with DEMs derived from EVE. This result further validates the corrections we derived from comparison with EVE.

Excluding the cooler contributions in the 94 and $131 \AA$ response functions, the only way to explain the observed signal in the 94 and 131 channels would be to assume that a substantial amount of hot $(T>6 \mathrm{MK})$ plasma exists throughout the corona. The most significant impact of the modification to the temperature response functions is the suppression of spurious hot tails on the inferred DEM distributions. 
Figure 12 Hinode/EIS and XRT were used to derive DEMs for the sub-regions shown in Figure 5; those DEMs are fairly similar to those obtained with the six AIA Fe channels alone in the temperature range where most of the AIA emission is formed.

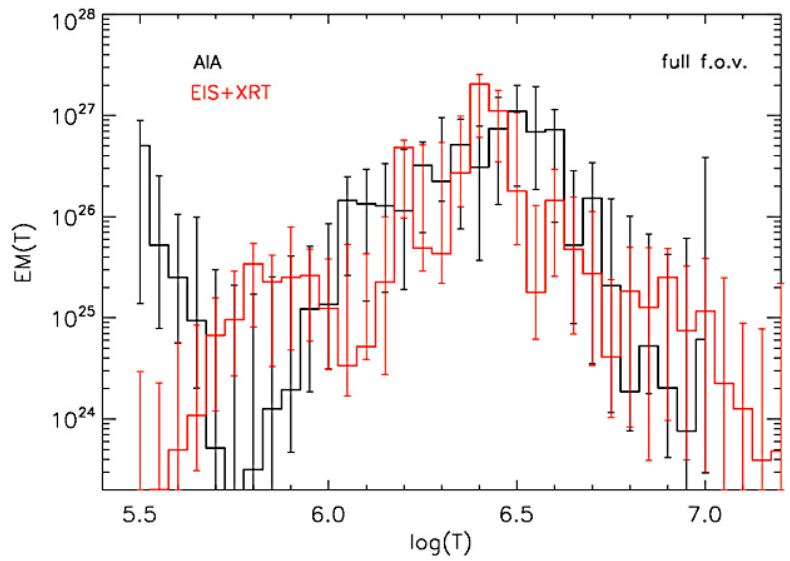

\subsection{DEMs with AIA and EIS + XRT}

A secondary benefit of ensuring accurate photometric calibration is that it allows us to leverage observations from multiple instruments. Combining AIA data with observations from EIS, as in Warren, Brooks, and Winebarger (2011), makes it possible to measure temperatures with finer coverage and resolution than with AIA alone, and to take advantage of the diagnostic line ratios in the EIS data set. Adding in data from Hinode/XRT allows an even more detailed insight, in particular by constraining the high-temperature end of the temperature distribution (Winebarger et al., 2011).

Using the observations from Figure 5, we fit DEMs for the sub-regions identified in Table 2 using data from AIA alone and with a combination of EIS and XRT. The results are shown in Figure 12. As expected, the combination of the large number of EUV lines from EIS and the high-temperature constraint from XRT provides the most complete temperature constraint; however, the agreement between the AIA-only DEM and the one obtained from EIS and XRT is reasonably good, especially within the temperature range from 1-4 MK where the AIA channels are most sensitive.

To further validate the modifications to the 94 and $131 \AA$ response functions, we then used the DEM inferred from EIS and XRT observations to predict AIA count rates using both the original and the modified temperature response functions. The results are shown in Figure 13. Once again, the agreement in the 94 and $131 \AA$ channels is dramatically improved with the revised functions. Moreover, the fact that the EIS/XRT-derived DEM agrees as well as it does with the AIA observations emphasizes that the apparent fine-scale discrepancies between the DEMs shown in Figure 12 are not necessarily significant. AIA data alone would not reject a DEM solution such as the one produced with EIS and XRT.

\section{Conclusions}

The photometric calibration of SDO/AIA as a function of wavelength generally agrees well with SDO/EVE, Hinode/EIS, and SORCE/SOLSTICE. If we assume that the calibration of EVE is correct, we can correct for residual errors in the AIA calibration and ongoing changes in the instrument sensitivity by normalizing the AIA wavelength response functions using EVE observations. However, there is still some uncertainty in the shape of the $335 \AA$ passband, which cannot be corrected for with a simple normalization. 
Figure 13 The DEMs obtained with EIS and XRT (see Figure 12) were then folded through the AIA temperature response functions to predict count rates for those regions. The observed AIA count rates agree better with those predicted using the modified response functions for the 94 and $131 \AA$ channels.

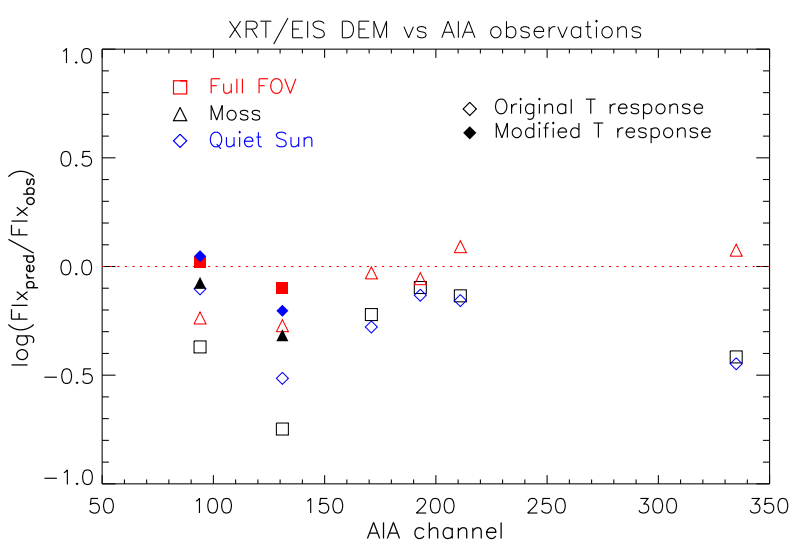

The determination of the instrument response as a function of temperature is limited by the deficiency of the CHIANTI database in the 50-170 $\AA$ wavelength range; however, pending improvements to CHIANTI, we propose an empirical correction to the temperature response functions of the 94 and $131 \AA$ channels that produces good agreement with DEM models obtained from other sources.

These improvements to the accuracy of the AIA response functions allow a more accurate quantitative analysis of the data obtained by AIA.

Acknowledgements The authors thank the members of the EVE team for providing helpful advice and excellent data. Hinode is a Japanese mission developed and launched by ISAS/JAXA, with NAOJ as domestic partner and NASA and STFC (UK) as international partners. It is operated by these agencies in co-operation with ESA and NSC (Norway). This work is supported by NASA under contract NNG04EA00C.

Open Access This article is distributed under the terms of the Creative Commons Attribution License which permits any use, distribution, and reproduction in any medium, provided the original author(s) and the source are credited.

\section{References}

Aschwanden, M.J., Boerner, P.: 2011, Solar corona loop studies with the atmospheric imaging assembly. I. Cross-sectional temperature structure. Astrophys. J. 732, 81.

Boerner, P., Edwards, C., Lemen, J., Rausch, A., Schrijver, C., Shine, R., Shing, L., Stern, R., Tarbell, T., Title, A., Wolfson, C.J., Soufli, R., Spiller, E., Gullikson, E., McKenzie, D., Windt, D., Golub, L., Podgorski, W., Testa, P., Weber, M.: 2012, Initial calibration of the Atmospheric Imaging Assembly (AIA) on the Solar Dynamics Observatory (SDO). Solar Phys. 275, 41 -66.

Culhane, J.L., Harra, L.K., James, A.M., Al-Janabi, K., Bradley, L.J., Chaudry, R.A., Rees, K., Tandy, J.A., Thomas, P., Whillock, M.C.R., Winter, B., Doschek, G.A., Korendyke, C.M., Brown, C.M., Myers, S., Mariska, J., Seely, J., Lang, J., Kent, B.J., Shaughnessy, B.M., Young, P.R., Simnett, G.M., Castelli, C.M., Mahmoud, S., Mapson-Menard, H., Probyn, B.J., Thomas, R.J., Davila, J., Dere, K., Windt, D., Shea, J., Hagood, R., Moye, R., Hara, H., Watanabe, T., Matsuzaki, K., Kosugi, T., Hansteen, V., Wikstol, Ø.: 2007, The EUV Imaging Spectrometer for Hinode. Solar Phys. 243, 19-61.

Del Zanna, G.: 2012, Benchmarking atomic data for astrophysics: a first look at the soft x-ray lines. Astron. Astrophys. 546, A97.

Del Zanna, G., Storey, P.J., Badnell, N.R., Mason, H.E.: 2012, Atomic data for astrophysics: Fe X soft x-ray lines. Astron. Astrophys. 541, A90.

Dere, K.P., Landi, E., Mason, H.E., Monsignori Fossi, B.C., Young, P.R.: 1997, CHIANTI - An atomic database for emission lines. Astron. Astrophys. Suppl. 125, 149-173. 
Dere, K.P., Moses, J.D., Delaboudinière, J.P., Brunaud, J., Carabetian, C., Hochedez, J.F., Song, X.Y., Catura, R.C., Clette, F., Defise, J.M.: 2000, The preflight photometric calibration of the Extreme-ultraviolet Imaging Telescope EIT. Solar Phys. 195, $13-44$.

Freeland, S.L., Handy, B.N.: 1998, Data analysis with the SolarSoft system. Solar Phys. 182, 497-500.

Golub, L., Deluca, E., Austin, G., Bookbinder, J., Caldwell, D., Cheimets, P., Cirtain, J., Cosmo, M., Reid, P., Sette, A., Weber, M., Sakao, T., Kano, R., Shibasaki, K., Hara, H., Tsuneta, S., Kumagai, K., Tamura, T., Shimojo, M., McCracken, J., Carpenter, J., Haight, H., Siler, R., Wright, E., Tucker, J., Rutledge, H., Barbera, M., Peres, G., Varisco, S.: 2007, The X-Ray Telescope (XRT) for the Hinode mission. Solar Phys. 243, $63-86$.

Guennou, C., Auchère, F., Soubrié, E., Bocchialini, K., Parenti, S., Barbey, N.: 2012, On the accuracy of the differential emission measure diagnostics of solar plasmas. Application to SDO/AIA. II. Multithermal plasmas. Astrophys. J. Suppl. 203, 26.

Handy, B.N., Acton, L.W., Kankelborg, C.C., Wolfson, C.J., Akin, D.J., Bruner, M.E., Caravalho, R., Catura, R.C., Chevalier, R., Duncan, D.W., Edwards, C.G., Feinstein, C.N., Freeland, S.L., Friedlaender, F.M., Hoffmann, C.H., Hurlburt, N.E., Jurcevich, B.K., Katz, N.L., Kelly, G.A., Lemen, J.R., Levay, M., Lindgren, R.W., Mathur, D.P., Meyer, S.B., Morrison, S.J., Morrison, M.D., Nightingale, R.W., Pope, T.P., Rehse, R.A., Schrijver, C.J., Shine, R.A., Shing, L., Strong, K.T., Tarbell, T.D., Title, A.M., Torgerson, D.D., Golub, L., Bookbinder, J.A., Caldwell, D., Cheimets, P.N., Davis, W.N., Deluca, E.E., McMullen, R.A., Warren, H.P., Amato, D., Fisher, R., Maldonado, H., Parkinson, C.: 1999, The transition region and coronal explorer. Solar Phys. 187, 229-260.

Hock, R.A., Eparvier, F.G.: 2008, Cross-calibration of TIMED SEE and SOHO EIT irradiances. Solar Phys. 250, $207-219$.

Hock, R.A., Chamberlin, P.C., Woods, T.N., Crotser, D., Eparvier, F.G., Woodraska, D.L., Woods, E.C.: 2012, Extreme ultraviolet Variability Experiment (EVE) Multiple EUV Grating Spectrographs (MEGS): Radiometric calibrations and results. Solar Phys. 275, $145-178$.

Landi, E., Young, P.R.: 2010, The relative intensity calibration of Hinode/EIS and SOHO/SUMER. Astrophys. J. 714, 636-643.

Landi, E., Del Zanna, G., Young, P.R., Dere, K.P., Mason, H.E.: 2012, CHIANTI - An atomic database for emission lines. XII. Version 7 of the database. Astrophys. J. 744, 99.

Lemen, J.R., Title, A.M., Akin, D.J., Boerner, P.F., Chou, C., Drake, J.F., Duncan, D.W., Edwards, C.G., Friedlaender, F.M., Heyman, G.F., Hurlburt, N.E., Katz, N.L., Kushner, G.D., Levay, M., Lindgren, R.W., Mathur, D.P., McFeaters, E.L., Mitchell, S., Rehse, R.A., Schrijver, C.J., Springer, L.A., Stern, R.A., Tarbell, T.D., Wuelser, J.P., Wolfson, C.J., Yanari, C., Bookbinder, J.A., Cheimets, P.N., Caldwell, D., Deluca, E.E., Gates, R., Golub, L., Park, S., Podgorski, W.A., Bush, R.I., Scherrer, P.H., Gummin, M.A., Smith, P., Auker, G., Jerram, P., Pool, P., Soufli, R., Windt, D.L., Beardsley, S., Clapp, M., Lang, J., Waltham, N.: 2012, The Atmospheric Imaging Assembly (AIA) on the Solar Dynamics Observatory (SDO). Solar Phys. 275, 17-40.

Liu, W., Title, A.M., Zhao, J., Ofman, L., Schrijver, C.J., Aschwanden, M.J., De Pontieu, B., Tarbell, T.D.: 2011 , Direct imaging of quasi-periodic fast propagating waves of $\sim 2000 \mathrm{~km} \mathrm{~s}^{-1}$ in the low solar corona by the Solar Dynamics Observatory Atmospheric Imaging Assembly. Astrophys. J. Lett. 736, L13.

McClintock, W.E., Rottman, G.J., Woods, T.N.: 2005, Solar-stellar irradiance comparison experiment II (SOLSTICE II): instrument concept and design. Solar Phys. 230, 225 - 258.

Schrijver, C.J., Aulanier, G., Title, A.M., Pariat, E., Delannée, C.: 2011, The 2011 February 15 X2 flare, ribbons, coronal front, and mass ejection: interpreting the three-dimensional views from the solar dynamics observatory and STEREO guided by magnetohydrodynamic flux-rope modeling. Astrophys. J. 738, 167.

Shine, R.A., Nightingale, R.W., Boerner, P., Tarbell, T.D., Wolfson, C.J.: 2010, Flat fielding and image alignments for AIA/SDO data images. AGU Fall Meeting, SH23C-1872.

Teriaca, L., Warren, H.P., Curdt, W.: 2012, Spectroscopic observations of Fe XVIII in solar active regions. Astrophys. J. Lett. 754, L40.

Testa, P., Drake, J.J., Landi, E.: 2012, Testing EUV/X-ray atomic data for the Solar Dynamics Observatory. Astrophys. J. 745, 111.

Vernazza, J.E., Reeves, E.M.: 1978, Extreme ultraviolet composite spectra of representative solar features. Astrophys. J. Suppl. 37, 485.

Wang, T., Thomas, R.J., Brosius, J.W., Young, P.R., Rabin, D.M., Davila, J.M., Del Zanna, G.: 2011, Underflight calibration of SOHO/CDS and Hinode/EIS with EUNIS-07. Astrophys. J. Suppl. 197, 32.

Warren, H.P., Brooks, D.H., Winebarger, A.R.: 2011, Constraints on the heating of high-temperature active region loops: observations from Hinode and the Solar Dynamics Observatory. Astrophys. J. 734, 90.

Warren, H.P., O’Brien, C.M., Sheeley, N.R.: 2011, Observations of reconnecting flare loops with the Atmospheric Imaging Assembly. Astrophys. J. 742, 92. 
Winebarger, A.R., Schmelz, J.T., Warren, H.P., Saar, S.H., Kashyap, V.L.: 2011, Using a differential emission measure and density measurements in an active region core to test a steady heating model. Astrophys. J. 740, 2.

Woods, T.N., Hock, R., Eparvier, F., Jones, A.R., Chamberlin, P.C., Klimchuk, J.A., Didkovsky, L., Judge, D., Mariska, J., Warren, H., Schrijver, C.J., Webb, D.F., Bailey, S., Tobiska, W.K.: 2011, New solar extreme-ultraviolet irradiance observations during flares. Astrophys. J. 739, 59.

Woods, T.N., Eparvier, F.G., Hock, R., Jones, A.R., Woodraska, D., Judge, D., Didkovsky, L., Lean, J., Mariska, J., Warren, H., McMullin, D., Chamberlin, P., Berthiaume, G., Bailey, S., Fuller-Rowell, T., Sojka, J., Tobiska, W.K., Viereck, R.: 2012, Extreme ultraviolet Variability Experiment (EVE) on the Solar Dynamics Observatory (SDO): Overview of science objectives, instrument design, data products, and model developments. Solar Phys. 275, 115. 